\title{
PARAMETER STUDY OF STABLE WALKING GAITS FOR NAO HUMANOID ROBOT
}

\author{
N. Latif A. Shaari ${ }^{1}$, Muhammad Razmi Bin Razali ${ }^{2}$, M. F. Miskon ${ }^{3}$, Ida S.Md Isa ${ }^{4}$ \\ ${ }^{1}$ Mechatronic Department, Universiti Teknikal Malaysia Melaka, Melaka, Malaysia,latifazyze@utem.edu.my \\ ${ }^{2}$ Universiti Teknikal Malaysia Melaka, Melaka, Malaysia, amie_joecole@yahoo.com \\ ${ }^{3}$ Mechatronic Department, Universiti Teknikal Malaysia Melaka, Melaka, Malaysia,fahmimiskon@utem.edu.my \\ ${ }^{4}$ Telecomunication Department, Universiti Teknikal Malaysia Melaka, Melaka, Malaysia, idasyafiza@utem.edu.my
}

\begin{abstract}
It is a challenge to any researcher to maintain the stability of the robot while in the walking motion. This paper proposes for parameter study of a walking pattern method which is inspired by the Dip Goswami (2009). The walking pattern is generated based on three points. These points are located at ankle left and right and one at the hip of the NAO humanoid robot. By using these points the walking gaits are generated to use as a reference point while walking motion. Then, an inverse kinematics with geometric solution of a ten degree-of-freedom humanoid robot is formulated from hip until the ankle joint. By sampling period of time with ten this reference point is used to find the joint angle of each link. The NAO humanoid robot is built in with force resistive sensor (FSR) located under both feet are used to determine the walking stability by using force distributer concept. The zero moment point of the robot is calculated on the normalized value between FSR reading from right leg and left leg. The result shown based on the real time simulation environment by using Webots Robotic Software. A simulation result shows that a NAO humanoid robot successfully walks in stable condition by following five different walking parameter setting. The humanoid robot is stable if and only if the normalized value of the ZMP is between 1 and -1 .
\end{abstract}

Index Terms: Walking gaits, NAO humanoid robot, ZMP

\section{INTRODUCTION}

For a humanoid robot to perform a task successfully in a real world environment, stable dynamic biped locomotion is required. However, the humanoid robots nowadays still do not satisfy the aforementioned demands of the real and uncertain environment. Researches on the motion control of the humanoid robot still in the midway, whereas further development and improvement are required because the humanoid robot are extremely complex and non-linear dynamical system.

The human walking motion is a natural model to be addressed largely in kinematics of many humanoid robots. This objective is intended to move towards convenience and smoothness of human walking. However, although the natural human walking motion is not very constrained, it seeks comprehensive degrees of freedom (DOF) throughout the body. The descriptions and models shown in this paper are based on the kinematics of the legs only where inspired by Dip Goswami. Haghighi, H. R. E. et al. presents a human like walking trajectories for the feet and the hips in order to achieve smooth and continuous walking pattern but the newly designed mechanism is just for $8 \mathrm{DOF}$ humanoid robots.
A lot of work model humanoid robot and its walking pattern by using Inverted Pendulum Model (IPM) [2-3]. In this model, the whole body is replaced with a concentrated mass located at the center of mass (CoM). This model often utilized to generated walking gaits for two legged mechanism. Inverted pendulum model is useful for analysis the stability by computing the ZMP which is the point on the ground where the resultant of every moment is zero. However this method is difficult to implement in high degree-of-freedom of humanoid robot. Another recent method introduced by Kim [4] uses Convolution-Sum-based algorithms to derive the center of mass trajectory in real time from a given zero moment point trajectory. The convolution-sum method inherently tracks the zero moment point trajectory exactly and is based on a linear inverted-pendulum model with a point mass. However, the problem that arises with the convolution method is that it tracks the zero moment point trajectory exactly, but does not take the jerk into account. In the case of biped locomotion method, the walking gaits are based on the optimal walking parameters, which solve the inverse kinematics model. Moreover, the generated gait maintains the postural stability using system dynamics.

One of the methods of a biped walking pattern generation that were taken into consideration is the Biped Locomotion method proposed by Dip Goswami [1]. This method generates 
the gaits by means of generating joint-trajectories and controlling each joint for trajectory-tracking so as to mimic the human locomotion. For this reason, this paper present a walking pattern trajectory generation inspired by Dip Goswami (2009) and it is tested using NAO humanoid robot in WebotTM robotic simulator. This paper organized as follows. Section II discusses about the research method that considering five types of parameters. Section III discusses about simulation results and analysis after carried out three experiments. Finally, conclusion follows in section IV.

\section{WALKING METHOD}

Common to a human walk, humanoid walk also consist of two phases, single support phase and double support phase. At the start of a walk, both feet are in contact with the ground, which we called the double support phase. This phase will change into the single support phase as soon as one of the legs lifts off the ground. The leg that is lifted off then moves to a desired location, and the cycle repeats itself as both feet are in contact with the ground again. The motion of the walk can be seen from Fig -1.

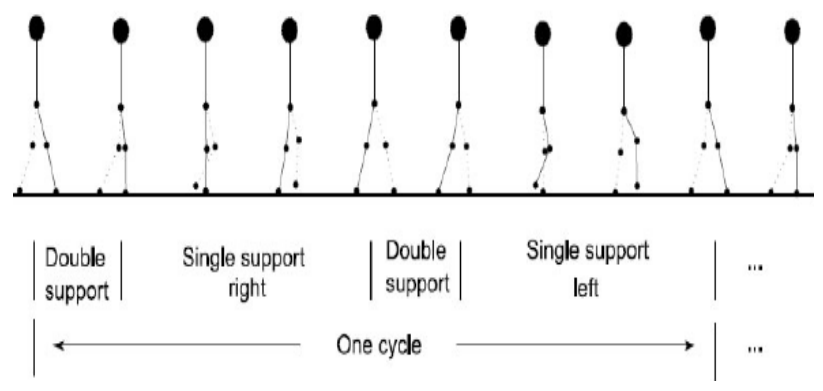

Fig -1: Phase of Walk
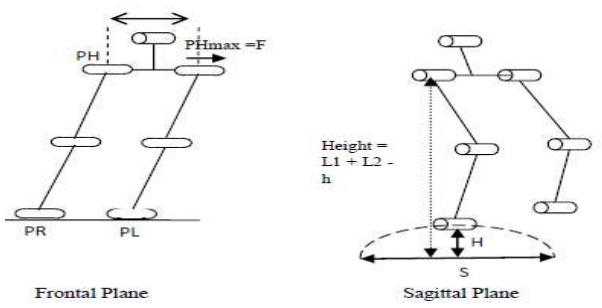

Fig -2: Gaits generation parameters.

Fig -2 shows the gaits generation parameter. This generated walking gaits consist of the following parameters, which are step-length is a length of the foot for one step (s), a bendingheight $(\mathrm{h})$ which is the constant height between the waist and ground during walking, a maximum lifting-height $(\mathrm{H})$ which is a foot maximum lifting height from the ground, a maximum frontal-shifted $(\mathrm{F})$ which is a maximum shift for the center of mass of the robot from the left and right side and period time
(T) which is the period between the left and right foot interchange.

The robot walking pattern is generated by selecting the suitable function of time for the three reference points, the stand leg at joint of left ankle coordinate (PL), the second point at joint of hip coordinate $(\mathrm{PH})$ and the third point at right ankle coordinate (PR).

For $0 \leq \mathrm{t} \leq \mathrm{T}$, PL will be static and acts as a reference for $\mathrm{PH}$ and $\mathrm{PR}$. For $\mathrm{T} \leq \mathrm{t} \leq 2 \mathrm{~T}$, the positions for $\mathrm{PL}$ and $\mathrm{PR}$ are interchanged. PL, PH and PR are the reference points that be prompted to be selected. PL and PR are selected according to the desired leg movement for a specific activity [1]. Dip Goswami mentioned the height of the waist-link is kept constant during walking A walking cycle for $0 \leq \mathrm{t} \leq 2 \mathrm{~T}$, the PL and PR for straight walking are provided by (2.1), (2.2) and (2.3).

\section{Left Foot Point}

$$
\begin{aligned}
& P L x(t)=\left(\frac{s}{2}\right) \sin \left(\frac{\pi}{T}\left(t-\frac{T}{2}\right)\right)(u(t-2 T)-u(t-T)) \\
& P L y(t)=-w(u(t-2 T)-u(t-T)) \\
& P L z(t)=H \sin \left(\pi\left(\frac{P L x(t)}{s}+0.5\right)\right)(u(t-2 T)-u(t-T))
\end{aligned}
$$

Right Foot Point

$$
\begin{aligned}
& P R x(t)=\left(\frac{s}{2}\right) \sin \left(\frac{\pi}{T}\left(t-\frac{T}{2}\right)\right)(u(t)-u(t-T)) \\
& P R y(t)=w(u(t)-u(t-T)) \\
& P R z(t)=H \sin \left(\pi\left(\frac{\operatorname{PRx}(t)}{s}+0.5\right)\right)(u(t)-u(t-T))
\end{aligned}
$$

Where s, w and $\mathrm{H}$ as shown in the Figure 2.The ${ }^{u(t)}$ is a unit step function given by equation (2.3).

$$
u(t)= \begin{cases}1 & \text { for } t \geq 0 \\ 0 & \text { for } t<0\end{cases}
$$

PH for straight walking is given by (2.4) and (2.5).

Hip Point

$$
\begin{aligned}
& P H x(t)=\left(\frac{s}{4}\right) \sin \left(\frac{\pi}{T}\left(\tau-\frac{T}{2}\right)\right) \\
& P H y(t)=F \sin \left(\frac{\pi}{2}\left(\sin \left(\frac{\tau \pi}{2 T}\right)+1\right)\right) \sin \left(\frac{\pi t}{T}\right) \\
& P H z(t)=L_{1}+L_{2}-h
\end{aligned}
$$


Where $\tau$ represents when

$$
\begin{array}{ll}
0 \leq t \leq T & \tau=t \\
T \leq t \leq 2 T & \tau=T-t
\end{array}
$$

Inverse Kinematic (IK) is used to calculate the joint angle by a given position. The PL and PR points will act as input position IK computation in Cartesian coordinate. Fig -3 shows the parameter of the inverse kinematic computation. Table -1 provide the parameters for the NAO humanoid robot.
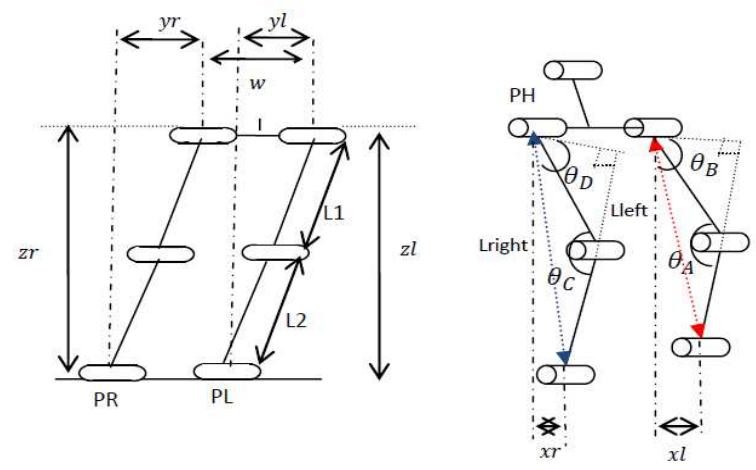

Fig -3: Inverse Kinematic parameters from hip to ankle.

Based on Fig -3, the trigonometric function is used to determine the angle of each joint. The distance from ankle position and the reference point, $\mathrm{PH}$ are used as the inputs for the trigonometric function. Next, the inverse kinematic algorithm will be described.

\section{Table -1: NAO HUMANOID ROBOT PARAMETER}

\begin{tabular}{|l|l|}
\hline Parameter & Values \\
\hline L1 (Tibia Length) & 0.1 meter \\
\hline L2 (Thigh Length) & 0.1 meter \\
\hline W & 0.1 meter \\
\hline
\end{tabular}

Distance between ankle and reference point $\mathrm{PH}$ for left and right leg are:

$$
\begin{array}{ll}
x r=P R x-P H x & x l=P L x-P H x \\
y r=-P H y & y l=-P H y \\
z r=P R z-P H z & z l=P L z-P H z \\
\text { Lright }=\sqrt{x r^{2}+y r^{2}+z r^{2}} \\
\text { Lleft }=\sqrt{x l^{2}+y l^{2}+z l^{2}}
\end{array}
$$

Then, the initial angle for left and right foot can be calculated as:

$$
\begin{aligned}
& \theta_{A}=\frac{\cos ^{-1}\left(L_{1}^{2}+L_{2}^{2}-\text { Lleft }^{2}\right)}{2 L_{1} L_{2}} \\
& \theta_{B}=\cos ^{-1}\left(\frac{L_{1} \sin \left(\theta_{A}\right)}{L_{1}}\right) \\
& \theta_{C}=\frac{\cos ^{-1}\left(L_{1}^{2}+L_{2}^{2}-\text { Lright }^{2}\right)}{2 L_{1} L_{2}} \\
& \theta_{D}=\cos ^{-1}\left(\frac{L_{1} \sin \left(\theta_{C}\right)}{L_{1}}\right)
\end{aligned}
$$

Where $\theta_{A}$ and $\theta_{B}$ will represent the angle for the left leg and the $\theta_{C}$ and $\theta_{D}$ represent the angle for the right leg.

NAO humanoid robot has a total of 25 degrees of freedom (DOF). The lower body part consist of 11 DOF includes legs and pelvis and the upper body part consist of 14 DOF includes trunk, arms and head. In this paper, only the lower body part will be used to test and verify the walking pattern generation. Each leg consists of 2 DOF at the ankle, 1 DOF at the knee and 2 DOF at the hip.
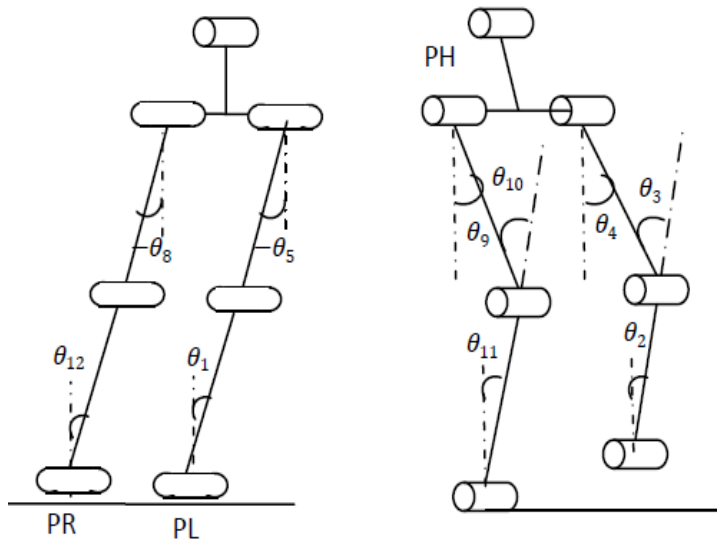

Fig -4: Joint angle parameters

Based on the walking pattern generation algorithm, the robot will walk approximately in a straight direction. The z-axis rotations at the hip joint are assume constant. Next, the joint angles for each link are determined.

The hip lateral angle and ankle lateral angle are calculated as:

$$
\begin{aligned}
& \theta_{1}=\tan ^{-1}\left(\frac{y l}{z l}\right) \\
& \theta_{5}=-\theta_{1} \\
& \theta_{12}=\tan ^{-1}\left(\frac{y r}{z r}\right) \\
& \theta_{8}=-\theta_{12}
\end{aligned}
$$


The joint angle for both knee are calculated as:

$$
\begin{aligned}
& \theta_{3}=\pi-\theta_{A} \\
& \theta_{10}=\pi-\theta_{C}
\end{aligned}
$$

Then, to calculate the hip joint in $\mathrm{x}$-axis, the equation of (2.8) is used for the left leg and equation of (2.9) is used for the right leg:

$$
\begin{aligned}
& \theta_{4}=\left(\frac{\pi}{2}\right)-\theta_{A}+\theta_{B}+\sin ^{-1}\left(\frac{x l}{\text { Lleft }}\right) \\
& \theta_{9}=\left(\frac{\pi}{2}\right)-\theta_{C}+\theta_{D}+\sin ^{-1}\left(\frac{x r}{\text { Lright }}\right)
\end{aligned}
$$

Finally, the last angles at the ankle joint in $\mathrm{x}$-axis:

$$
\begin{aligned}
& \theta_{2}=\theta_{3}-\theta_{4} \\
& \theta_{11}=\theta_{10}-\theta_{11}
\end{aligned}
$$

A discussion of the Zero Moment Point (ZMP) concept is based on a FSR (Force Sensitive Resistors) which is used to measure the trajectory of the walking pattern of the NAO humanoid robot walking. Generally, the total moment of the force on the point is equal to zero.

In the simulation, all four FSR sensors are place under each foot of the NAO humanoid robot as shown in Fig -6. The fundamental of ZMP computation and implementation are described in $[1,5]$. In walking sequence, when one leg is swinging, the mass of the whole body of the robot is connected to the stance foot which are same as inverted pendulum model.

Table -2: FSR POSITIONS IN THE ANKLE FRAME

\begin{tabular}{|l|l|l|}
\hline FSR Name & $\begin{array}{l}\text { Position X (m) } \\
\text { [Ankle Frame] }\end{array}$ & $\begin{array}{l}\text { Position Y (m) } \\
\text { [Ankle Frame] }\end{array}$ \\
\hline LFsrFL & 0.07025 [LEFT] & 0.0299 [LEFT] \\
\hline LFsrFR & $0.07025[$ LEFT] & $-0.0231[$ LEFT] \\
\hline LFsrRL & $-0.03025[$ LEFT] & $0.0299[$ LEFT] \\
\hline LFsrRR & $-0.02965[$ LEFT] & $-0.0191[$ LEFT] \\
\hline RFsrFL & $0.07025[$ RIGHT] & $0.0231[$ RIGHT] \\
\hline RFsrFR & $0.07025[$ RIGHT] & -0.0299 [RIGHT] \\
\hline RFsrRL & $-0.03025[$ RIGHT] & 0.0191 [RIGHT] \\
\hline RFsrRR & -0.02965 [RIGHT] & -0.0299 [RIGHT] \\
\hline
\end{tabular}
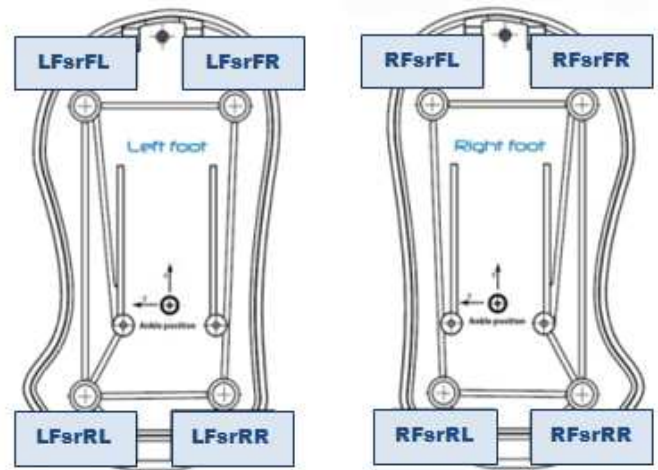

Fig -5: Force Sensitive Resistor position for NAO humanoid robot

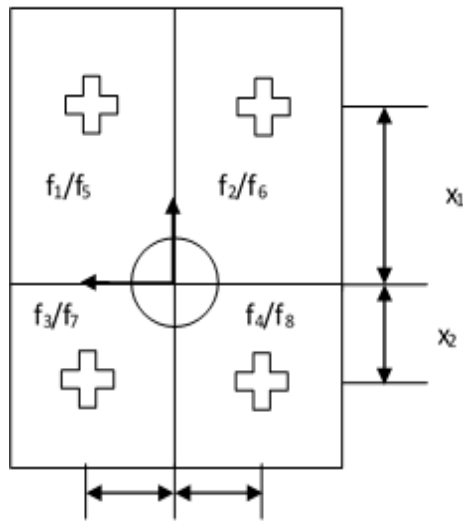

$\mathrm{y}_{1} \quad \mathrm{y}_{2}$

Fig -6: FSR place at both left and right feet.

The ZMP point in a sagittal plane XZMP and frontal plane for YZMP point based on force sensors shown in Fig -7 From this figure, the point $\mathrm{C}$ is a center of mass of the humanoid robot and point $\mathrm{O}$ is the ankle joint of the robot.
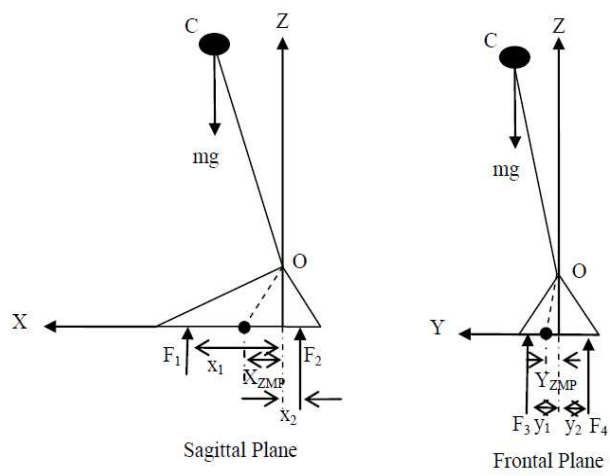

Fig -7: Simplified model of the ZMP point based on the FSR sensors. 
Then, based on Fig - 6 and Fig -7, the ZMP in the x-direction can be determined by:

$$
\begin{gathered}
F_{1}\left(x_{1}-x_{z m p}\right)-F_{2}\left(x_{2}+x_{z m p}\right)=0 \\
\left(F_{1}+F_{2}\right) x_{z m p}=F_{1} x_{1}-F_{2} x_{2} \\
x_{z m p}=\frac{F_{1} x_{1}-F_{2} x_{2}}{F_{1}+F_{2}}
\end{gathered}
$$

Where $F_{1}$ and $F_{2}$ are the sum of the FSR sensor in the sagittal plane.

$$
\left.\begin{array}{l}
F_{1}=f_{1}+f_{2} \\
F_{2}=f_{3}+f_{4}
\end{array}\right\} \text { Left Foot }
$$

and

$$
\left.\begin{array}{l}
F_{1}=f_{5}+f_{6} \\
F_{2}=f_{7}+f_{8}
\end{array}\right\} \text { Right Foot }
$$

During the single support phase, a distance of $\mathrm{x} 1$ and $\mathrm{x} 2$ are showed as in Fig -6 . Then, the ZMP point in y-direction can be determined as:

$$
\begin{gathered}
F_{3}\left(y_{1}-y_{z m p}\right)-F_{4}\left(y_{2}-y_{z m p}\right)=0 \\
\left(F_{3}+F_{4}\right) y_{z m p}=F_{3} y_{1}-F_{4} y_{2} \\
y_{z m p}=\frac{F_{3} y_{1}-F_{4} y_{2}}{F_{3}+F_{4}}
\end{gathered}
$$

Where the value of $F_{3}$ and $F_{4}$ can be get from the equation (2.16) and a distance y1 and y 2 as shown in Fig -6 .

$$
\left.\begin{array}{l}
F_{3}=f_{1}+f_{3} \\
F_{4}=f_{2}+f_{4}
\end{array}\right\} \text { Left Foot }
$$

And

$$
\left.\begin{array}{l}
F_{3}=f_{5}+f_{7} \\
F_{4}=f_{6}+f_{8}
\end{array}\right\} \text { Right Foot }
$$

The ZMP point will be interchange from left to right based on the COM movement. So, the normalized value of the distributing the ZMP point can be calculated as equation (2.17). This value can be used to plot a graph for determine the stability of the robot during walking between 1 and -1 .

$$
X_{Z M P}=\frac{\text { Left }_{z m p x}-\text { Right }_{z m p x}}{L e f t_{z m p x}+R i g h t_{z m p x}}
$$

$$
Y_{Z M P}=\frac{L e f t_{z m p y}-R i g h t_{z m p y}}{L e f t_{z m p y}+R i g h t_{z m p y}}
$$

\section{SIMULATION RESULT AND ANALYSIS}

From equation (2.1, 2.2 and 2.4) there are four parameter need to be consider which are bending height, frontal shift, step length and maximum foot lifting height. Three experiments were carried out to choose a walking trajectory parameter. Experiment 1 is a comparison between differences bending height. Then on experiment 2 is a comparison with difference frontal shift. Lastly on experiment 3 is a comparison between differences step length. Parameter setting in comparing the difference bending height is shown in Table -3 .

Table -3: Comparison with Different Bending Height

\begin{tabular}{|l|l|l|l|}
\hline $\begin{array}{l}\text { Step length } \\
(\mathrm{m})\end{array}$ & $\begin{array}{l}\text { Bending } \\
\text { Height }(\mathrm{m})\end{array}$ & $\begin{array}{l}\text { Lifting } \\
\text { Height }(\mathrm{m})\end{array}$ & $\begin{array}{l}\text { Frontal } \\
\text { Shift }(\mathrm{m})\end{array}$ \\
\hline 0.1 & 0.021 & 0.028 & 0.055 \\
\hline 0.1 & 0.020 & 0.028 & 0.055 \\
\hline 0.1 & 0.019 & 0.028 & 0.055 \\
\hline 0.1 & 0.018 & 0.028 & 0.055 \\
\hline 0.1 & 0.017 & 0.028 & 0.055 \\
\hline
\end{tabular}

From Table -3 the simulation was carried out by using Webots simulation software for real time experiment. The normalized value for Zero Moment Point (ZMP) was used to measure the stability of the NAO humanoid robot during performing walking.

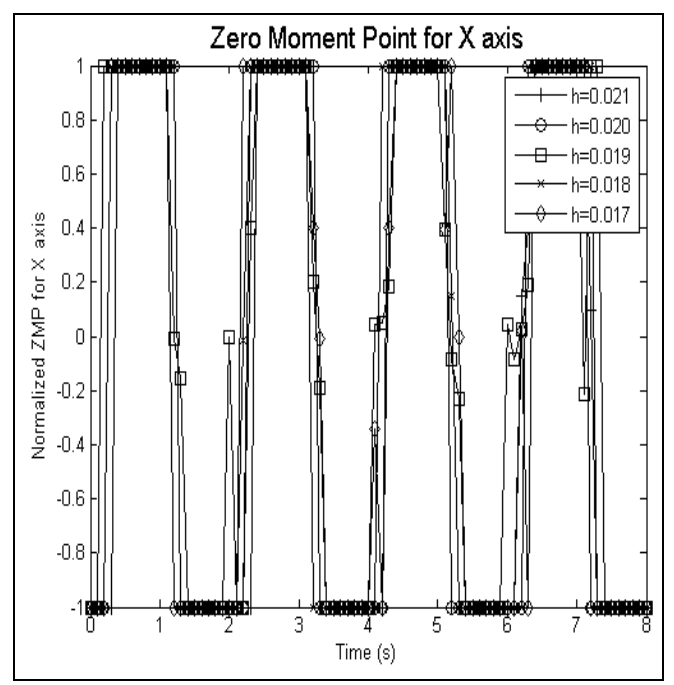

Chart -1: ZMP x-axis for different bending height

and 


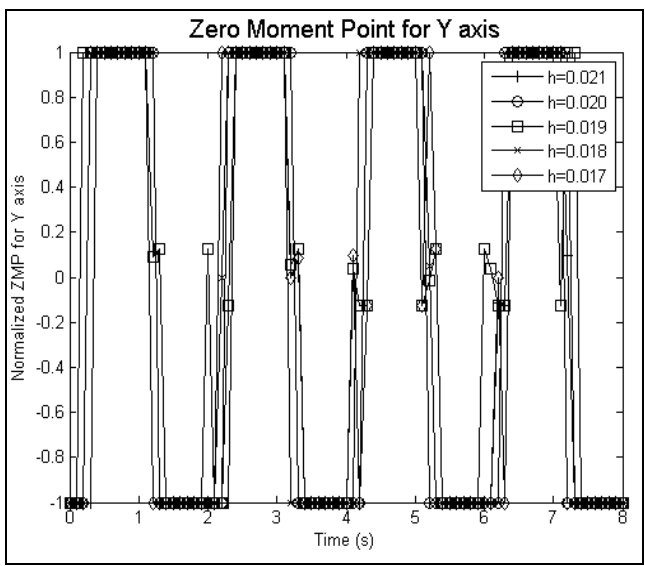

Chart -2: ZMP y-axis for different bending height

Chart -1 and chart -2 shows a normalized value for ZMP point for Sagittal plane (X-axis) and Frontal plane (Y-axis). By referring Chart -1 , the bending height for $\mathrm{h}=0.021 \mathrm{~m}$ until $\mathrm{h}=0.019 \mathrm{~m}$ and $\mathrm{h}=0.017 \mathrm{~m}$ show the normalized value for ZMP is fluctuating during interchange between left leg and right leg. This condition will cause the humanoid robot walking in unbalance. During the bending height equal to $0.018 \mathrm{~m}$ the graph show no fluctuates happen during transaction. So the best setting for bending height is $0.018 \mathrm{~m}$. In experiment 2 the value of front shift will compare and the value of bending height will use a result from experiment 1 . Table 4 shows a parameter setting in experiment 2.

Table -4: Comparison with different Frontal Shift, F

\begin{tabular}{|l|l|l|l|}
\hline $\begin{array}{l}\text { Step length } \\
(\mathrm{m})\end{array}$ & $\begin{array}{l}\text { Bending } \\
\text { Height }(\mathrm{m})\end{array}$ & $\begin{array}{l}\text { Lifting } \\
\text { Height }(\mathrm{m})\end{array}$ & $\begin{array}{l}\text { Frontal } \\
\text { Shift }(\mathrm{m})\end{array}$ \\
\hline 0.1 & 0.018 & 0.028 & 0.059 \\
\hline 0.1 & 0.018 & 0.028 & 0.057 \\
\hline 0.1 & 0.018 & 0.028 & 0.055 \\
\hline 0.1 & 0.018 & 0.028 & 0.053 \\
\hline 0.1 & 0.018 & 0.028 & 0.051 \\
\hline
\end{tabular}

The result of the experiment 2 is shown in Figure 9. Based on the graph shown in Chart -3 for ZMP $\mathrm{x}$-axis and Chart -4 for ZMP y-axis, when the frontal shift is equal to $0.055 \mathrm{~m}$ the robot can walked in stable condition. The purpose of the frontal shift when in single support phase, maximum frontalshifted, F involved in the transfer of ZMP points from one foot to the other foot. The robot maybe cannot lift the foot from the ground if the F value is too small. Thus, the maximum frontalshift, $\mathrm{F}$ is an important parameter to choose in conducting the walking robot experiment.

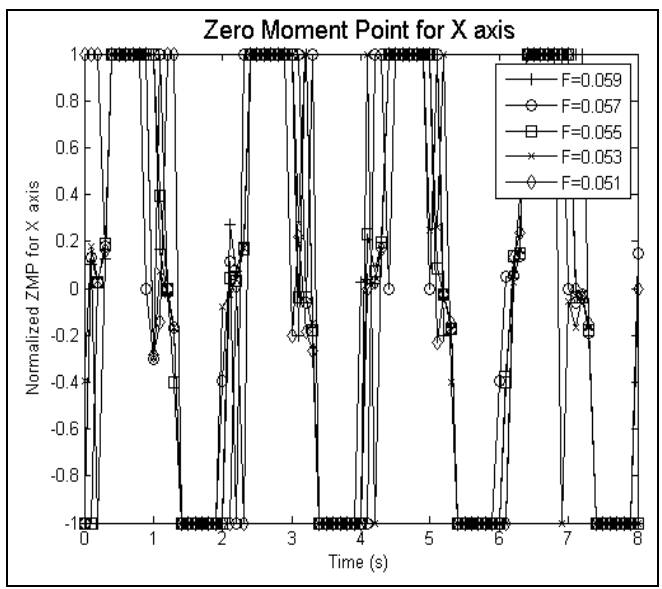

Chart -3: ZMP x-axis for different Frontal Shift

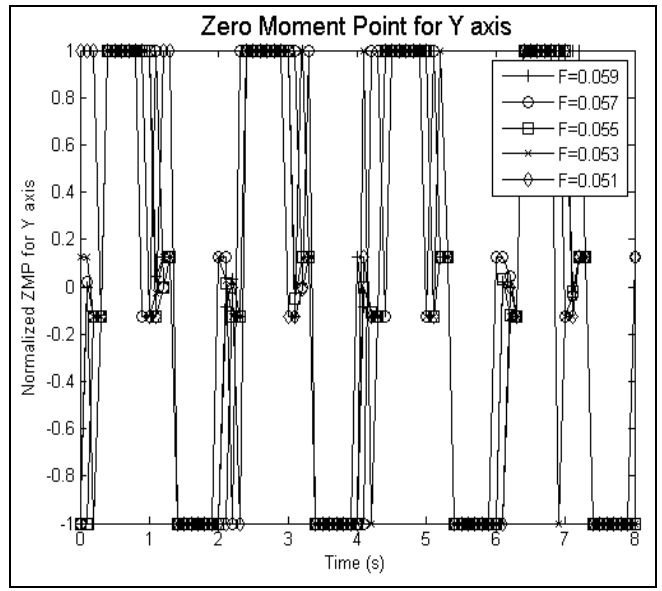

Chart -4: ZMP y-axis for different Frontal Shift

Lastly the experiment 3 is conducted by using result from experiment 1 and 2 . In experiment 3 the value of step length will vary between $0.08 \mathrm{~m}$ until $0.12 \mathrm{~m}$. This step length is important parameter to make sure the robot will walk smooth and no jerk. The parameter setting for experiment 3 shows in Table 5.

Table -5: Comparison with different Step Length, s

\begin{tabular}{|l|l|l|l|}
\hline $\begin{array}{l}\text { Step length } \\
(\mathrm{m})\end{array}$ & $\begin{array}{l}\text { Bending } \\
\text { Height }(\mathrm{m})\end{array}$ & $\begin{array}{l}\text { Lifting } \\
\text { Height }(\mathrm{m})\end{array}$ & $\begin{array}{l}\text { Frontal Shift } \\
(\mathrm{m})\end{array}$ \\
\hline 0.08 & 0.018 & 0.028 & 0.055 \\
\hline 0.09 & 0.018 & 0.028 & 0.055 \\
\hline 0.10 & 0.018 & 0.028 & 0.055 \\
\hline 0.11 & 0.018 & 0.028 & 0.055 \\
\hline 0.12 & 0.018 & 0.028 & 0.055 \\
\hline
\end{tabular}




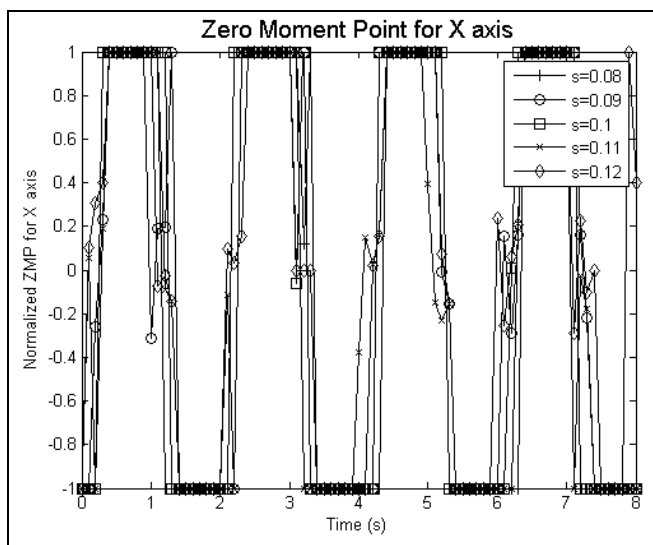

Chart -5: ZMP x-axis for different Step Length

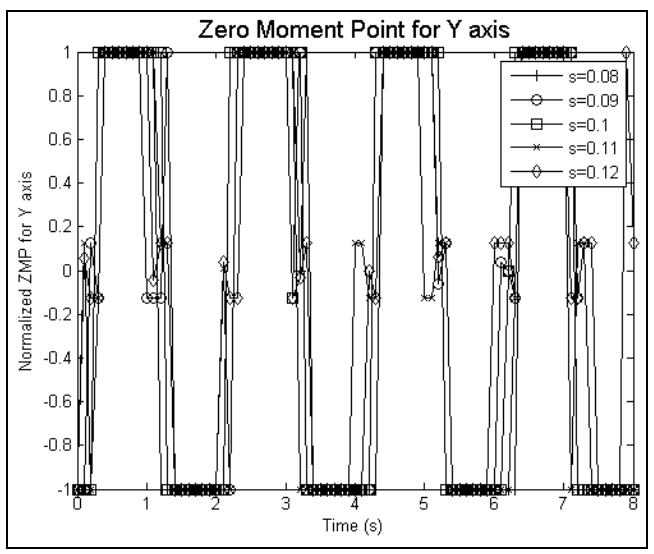

Chart -6: ZMP y-axis for different Step Length

Based on graph plotted in Chart -5 and -6 , the best step length is 0.1 which more stable when NAO humanoid robot performing walking motion. The step length will affect the robot walking speed and power consumption for a joint motor. Then the conclusion from the experiment conducted the best parameter setting for NAO humanoid robot is shown in Table -6 . The stability graph of the ZMP point is shown in Chart -6 . Only at one time the robot is unstable condition due to the real time data capture from simulation are effected by environment setting such as type of floor surface.

Table -6: Final Walking gait parameter setting

\begin{tabular}{|l|l|l|l|}
\hline $\begin{array}{l}\text { Step length } \\
(\mathrm{m})\end{array}$ & $\begin{array}{l}\text { Bending } \\
\text { Height }(\mathrm{m})\end{array}$ & $\begin{array}{l}\text { Lifting } \\
\text { Height }(\mathrm{m})\end{array}$ & $\begin{array}{l}\text { Frontal Shift } \\
(\mathrm{m})\end{array}$ \\
\hline 0.1 & 0.018 & 0.028 & 0.055 \\
\hline
\end{tabular}

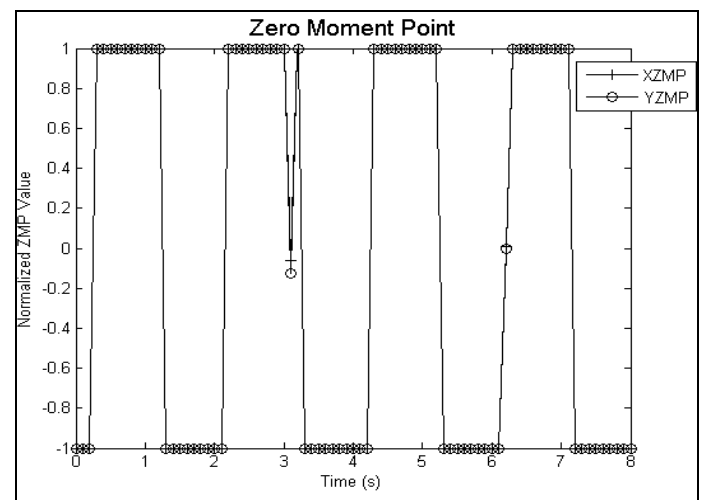

Chart -7: Normalized ZMP value

\section{CONCLUSIONS}

Throughout this experiment, the walking pattern generation considered ten degree of freedom (DOF) of the NAO humanoid robot. The walking parameters that were used are expressed in the inverse kinematic algorithm. The walking pattern is generated based on the 5 parameters. The parameters which are Period (T), Step Length (s), Bending Height (h), Lifting Height $(\mathrm{H})$ and Frontal Shift $(\mathrm{F})$ implemented in the walking pattern.

Stability of the robot during walking is measured by the ZMP trajectory points. Stability is an important factor in the course of experiment for a robot walking. Postural stability in humanoid robot walking has been a research challenge for more than three decades. The final walking pattern which has been performed in this experiment is not yet optimal. A trial and error procedure is needed to keep the robot from falling down. In the near future, offline tracking control methods will be used to generate a near optimal walking pattern which makes the robot walk straight and reduce the amount of time the robot falls while walking.

\section{ACKNOWLEDGEMENTS}

We would like to thank the Universiti Teknikal Malaysia Melaka (UTeM) by supporting a fund for making this project successful.

\section{REFERENCES}

[1]. Dip Goswami “ Biped Locomotion: Stability, Analysis, Gait Generator and Control" PHD Thesis, National University Singapore, 2009.

[2]. T. Sugihara, Y. Nakamura, and H. Inoue, "Real-time humanoid motion generation through ZMP manipulation based on inverted pendulum control" Proc. IEEE International Conference Robotics and Automation, vol. 2, pp. 1404-1409, May 2002.

[3]. Kyung-Kon Noh, Jin-Geol Kim and Uk-Youl Huh "Stability experiment of a biped walking robot with 
inverted pendulum" 30th IEEE Annual Conference of Industrial Electronics Society, pp. 2475 - 2479, vol. 3, 2-6 Nov. 2004

[4]. J.Kim, "Walking pattern generation of a biped walking robot using convolution sum," 7th IEEE-RAS International Conference on Humanoid Robots, pp. 539-544, Nov. 2007.

[5]. Tzuu-Hseng S. Li, Yu-Te Su, Shao-Hsien Liu, Jhen-Jia $\mathrm{Hu}$, and Ching-Chang Chen, Dynamic Balance Control for Biped Robot Walking Using Sensor Fusion, Kalman Filter, and Fuzzy Logic. IEEE 2009.

[6]. Vukobratovic, M., \&Borovac, B. "Zero-moment point thirty-five years of its life. International Journal of Humanoid Robotics", 1(1), 157-173., 2004

[7]. Shimojo, M., Araki, T., Ming, A., and Ishikawa M., " $A$ ZMP sensor for a biped robot" Proceedings. Conference on International Robotics and Automation (IEEE Cat. No. 06CH37729D), pages 1200-1205. 2006.

[8]. Christian Lathion, "Biped locomotion on the Hoap2 robot" Computer Science Master project, Biologically Inspired Robotics Group (BIRG), 2006.

[9]. S. Kajita, F. Kahehiro, K. Kaneko, K. Fujiwara, K. Harada, K. Yokoi, and H. Hirukawa, "Biped walking pattern generation using preview control of the zeromoment-point" Proc. IEEE International Conference of Robotics and Automation, vol. 2, pp. 1620-1626 Sep. 2003.

[10]. F. Yamasaki, K. Hosoda and M. Asada "An Energy consumption based control for humanoid walking" IEEE/RSJ International Conference on Intelligent Robots and System (IROS), pp. 2473 - 2477, vol. 3, 30 Sept.-5 Oct. 2002. 